\title{
Predictors of the effects of treatment for shoulder pain: protocol of an individual participant data meta-analysis
}

Danielle A. van der Windt ${ }^{*}$ (D), Danielle L. Burke ${ }^{1}$, Opeyemi Babatunde ${ }^{1}$, Miriam Hattle ${ }^{1}$, Cliona McRobert ${ }^{2}$, Chris Littlewood ${ }^{1}$ Gwenllian Wynne-Jones ${ }^{1}$, Linda Chesterton' ${ }^{1}$, Geert J. M. G. van der Heijden ${ }^{3}$, Jan C. Winters ${ }^{4}$, Daniel I. Rhon ${ }^{5}$, Kim Bennell ${ }^{6}$, Edward Roddy ${ }^{1,7}$, Carl Heneghan ${ }^{8}$, David Beard ${ }^{9}$, Jonathan L. Rees ${ }^{9}$ and Richard D. Riley ${ }^{1}$

\begin{abstract}
Background: Shoulder pain is one of the most common presentations of musculoskeletal pain with a 1-month population prevalence of between 7 and 26\%. The overall prognosis of shoulder pain is highly variable with $40 \%$ of patients reporting persistent pain 1 year after consulting their primary care clinician. Despite evidence for prognostic value of a range of patient and disease characteristics, it is not clear whether these factors also predict (moderate) the effect of specific treatments (such as corticosteroid injection, exercise, or surgery).

Objectives: This study aims to identify predictors of treatment effect (i.e. treatment moderators or effect modifiers) by investigating the association between a number of pre-defined individual-level factors and the effects of commonly used treatments on shoulder pain and disability outcomes.

Methods: This will be a meta-analysis using individual participant data (IPD). Eligible trials investigating the effectiveness of advice and analgesics, corticosteroid injection, physiotherapy-led exercise, psychological interventions, and/or surgical treatment in patients with shoulder conditions will be identified from systematic reviews and an updated systematic search for trials, and risk of bias will be assessed. Authors of all eligible trials will be approached for data sharing. Outcomes measured will be shoulder pain and disability, and our previous work has identified candidate predictors. The main analysis will be conducted using hierarchical one-stage IPD metaanalysis models, examining the effect of treatment-predictor interaction on outcome for each of the candidate predictors and describing relevant subgroup effects where significant interaction effects are detected. Random effects will be used to account for clustering and heterogeneity. Sensitivity analyses will be based on (i) exclusion of trials at high risk of bias, (ii) use of restricted cubic splines to model potential non-linear associations for candidate predictors measured on a continuous scale, and (iii) the use of a two-stage IPD meta-analysis framework.
\end{abstract}

Discussion: Our study will collate, appraise, and synthesise IPD from multiple studies to examine potential predictors of treatment effect in order to assess the potential for better and more efficient targeting of specific treatments for individuals with shoulder pain.

Systematic review registration: PROSPERO CRD42018088298

Keywords: Shoulder conditions, Pain, Disability, Individual participant data meta-analysis, Predictors of treatment effect

\footnotetext{
* Correspondence: d.van.der.windt@keele.ac.uk

${ }^{1}$ Institute for Primary Care and Health Sciences, Arthritis Research UK Primary

Care Centre, Centre for Prognosis Research, Keele University, Keele, UK

Full list of author information is available at the end of the article
}

(c) The Author(s). 2019 Open Access This article is distributed under the terms of the Creative Commons Attribution 4.0 International License (http://creativecommons.org/licenses/by/4.0/), which permits unrestricted use, distribution, and reproduction in any medium, provided you give appropriate credit to the original author(s) and the source, provide a link to the Creative Commons license, and indicate if changes were made. The Creative Commons Public Domain Dedication waiver (http://creativecommons.org/publicdomain/zero/1.0/) applies to the data made available in this article, unless otherwise stated. 


\section{Background}

Musculoskeletal conditions are globally among the leading causes of years lived with disability [1]. Shoulder pain is one of the most common presentations with a 1month population prevalence of between 7 and 26\% [2]. Annually, about $4 \%$ of adults will visit their general practitioner (GP) for shoulder pain [3, 4], resulting in approximately 1.5 million consultations in England. The total annual costs of shoulder pain to society have been estimated at $£ 100$ million for the UK [5]. Mean annual costs per patient have been estimated at $£ 3500$ (Sweden) [6] and $£ 8500$ (the USA) [7] per patient, with costs of surgical interventions and work absence contributing most to this estimate.

Systematic reviews (e.g. [8-14]) as well as recently published trials (e.g. [15-18]) consistently show moderate short-term effects of primary care interventions such as corticosteroid injection and exercise, but a lack of evidence for long-term (> 6 months) benefit. The rates of surgery in the UK increased by more than sevenfold (750\%) for subacromial decompression alone from 5.2/ 100,000 in $2001-2002$ to $40.2 / 100,000$ in $2009-2010$ [19], but there is increasing evidence that some surgical shoulder interventions are no more effective than nonsurgical treatments or placebo surgery [20-25]. This demonstrates an increase in the use of healthcare resources, without evidence of improvement in long-term patient outcomes, and little guidance to support optimal treatment and referral decisions. The implementation of more promising or better-targeted treatment for shoulder pain is hampered by a lack of high-quality evidence for the predictive value and clinical utility of diagnostic and prognostic information.

Clinicians use information from the patient's clinical history and physical examination to assess the severity and possible origin of the problem (rotator cuff tears or tendinopathy, subacromial bursitis, adhesive capsulitis/ frozen shoulder, acromioclavicular conditions, glenohumeral osteoarthritis), although diagnostic accuracy is limited [26-28]. Qualitative research shows inconsistency in the labelling of shoulder conditions and little impact of diagnostic labels on treatment decisions [29]. Based on their clinical assessment, clinicians decide which type of treatment (e.g. advice and pain relief only; exercise with or without mobilisation; corticosteroid injection; referral for a specialist (orthopaedic) opinion) is most suitable for their patient. There is little evidence, however, to support such decisions. The uncertainties clinicians experience in the management of shoulder pain and the different strategies they use to deal with these have been confirmed in a recent interview study among Dutch GPs [30] and in surveys conducted in Australia [31] and the UK [32].
The overall prognosis of shoulder pain is highly variable with $40-50 \%$ of patients reporting persistent pain 6-12 months after consulting their primary care clinician [33-36]. In addition to diagnostic information obtained from the clinical assessment, the long-term prognosis can be influenced by a range of factors, including age, educational level, symptom duration, previous episodes, disability scores, and multisite pain [35-40]. The prognostic value of psychological and social factors is less clear, but evidence suggests that fearavoidance beliefs, pain catastrophizing, depressive symptoms, low pain self-efficacy, and aspects of the psychosocial work environment (high job strain, low supervisor/co-worker support) are associated with poor long-term outcome [36, 38, 39, 41-46], particularly in those with chronic or recurrent shoulder pain $[47,48]$.

Qualitative research in patients with shoulder pain has emphasised the anxiety patients experience due to uncertainty about prognosis and treatment. Patients highlighted the lack of clear, written information about the condition and expressed a wish for faster, betterdefined treatment choices [49]. This was confirmed by results of a recently completed James Lind Alliance Priority Setting Partnership, with one of the top 10 research priorities being 'How can we ensure that patients see the right clinicians promptly and correctly, and does this lead to better outcomes?' [50] Despite evidence for prognostic value of a range of patient and disease characteristics, it is not clear whether some of these factors may also predict the effectiveness of specific treatments (such as corticosteroid injection, exercise, or surgery) in an individual or subgroups of similar individuals, i.e. modify or moderate the effect of treatment, and may inform decisions regarding the type of treatment likely to be most effective for specific (subgroups of) patients [51]. There is a clear need for research investigating individual-level predictors of treatment effect in order to assess the possibility of better and more efficient targeting of shoulder pain treatments, potentially improving patient outcomes.

\section{Study objectives}

Investigating treatment effect moderation requires data from randomised controlled trials in order to estimate candidate treatment-predictor interactions and describe subgroup effects. A single randomised trial is generally not powered to detect genuine individual-level predictors. To address this, we will collate and synthesise individual participant data (IPD) from multiple trials to increase the power to identify genuine predictors of treatment effect. Our aim is to determine moderation of the effects of four types of treatments that are most commonly used in the primary care management of painful shoulder conditions and where evidence regarding predictors of treatment effect would inform 
decisions regarding their optimal use: (a) corticosteroid injection, (b) physiotherapy-led exercise, (c) psychological treatments, and (d) surgical interventions on shoulder pain and disability outcomes by a limited number of a priori defined individual-level candidate predictors (patient characteristics). The IPD metaanalysis will be conducted in three steps with the following objectives:

1. To estimate the overall effects of each of these four treatments separately, when compared to a control intervention (no treatment, advice and analgesics only, or sham)

2. To estimate the relative effects when these interventions are directly compared against each other in pragmatic randomised trials

3. Within each of these comparisons separately, to estimate treatment-predictor interactions for a number of a priori defined patient or disease characteristics and describe relevant subgroup effects when interactions are detected

The results of the IPD meta-analysis will be used to define profiles of patients with shoulder pain likely to respond well to each of the treatments listed above. As part of a larger programme of research, the IPD metaanalysis will form the basis of the design of a treatment decision tool for patients consulting with shoulder pain in primary care, to be used as part of a stratified care intervention and tested against usual care for shoulder pain in a large pragmatic randomised controlled trial.

\section{Methods}

An a priori protocol was established for this IPD metaanalysis and registered with the international prospective register of systematic reviews: PROSPERO (www.crd. york.ac.uk/prospero/), number CRD42018088298.

\section{Eligibility criteria (see also Table 1) Design and setting}

IPD will be included from randomised clinical trials conducted in the community, primary healthcare, or secondary healthcare settings. No language restrictions will be used.

\section{Study population}

The IPD meta-analysis will include trials conducted in adult patients with general or non-specified shoulder

Table 1 Eligibility criteria for including trials in the IPD meta-analysis

\begin{tabular}{|c|c|c|}
\hline & Include & Exclude \\
\hline Design & $\begin{array}{l}\text { Randomised clinical trials, including individual and cluster-randomised trials, no } \\
\text { language restrictions }\end{array}$ & Any other non-randomised design \\
\hline Setting & Any healthcare setting & $\begin{array}{l}\text { Preventative intervention studies (e.g. in workplace } \\
\text { settings) }\end{array}$ \\
\hline Population & $\begin{array}{l}\text { - Adults (18 years and older) } \\
\text { - General or non-specified shoulder pain or diagnosed with (i) subacromial con- } \\
\text { dition (rotator cuff tendinopathy, rotator cuff tear, subacromial bursitis), (ii) frozen } \\
\text { shoulder/adhesive capsulitis, (iii) glenohumeral osteoarthritis, (iv) shoulder } \\
\text { instability. }\end{array}$ & $\begin{array}{l}\text { - Acute trauma (fractures, traumatic dislocations) } \\
\text { - Inflammatory arthritis (rheumatoid arthritis, } \\
\text { polymyalgia rheumatica) } \\
\text { - Shoulder pain resulting from cervical } \\
\text { radiculopathy } \\
\text { - Stroke-related shoulder pain }\end{array}$ \\
\hline Interventions & $\begin{array}{l}\text { - Corticosteroid injection } \\
\text { - Physiotherapy-led exercise (with or without manual therapy) } \\
\text { - Interventions addressing psychological factors (e.g. cognitive behavioural } \\
\text { approaches, multimodal interventions) } \\
\text { - Surgical interventions }\end{array}$ & $\begin{array}{l}\text { Other, less common treatment options, or } \\
\text { treatments with very limited evidence of } \\
\text { effectiveness }\end{array}$ \\
\hline Control & $\begin{array}{l}\text { - Advice and pain relief only } \\
\text { - Sham/placebo intervention } \\
\text { - Direct comparisons between the interventions listed above }\end{array}$ & $\begin{array}{l}\text { - Comparisons with other interventions } \\
\text { - Comparisons of different dosages, types, or modes } \\
\text { of delivery of the same intervention }\end{array}$ \\
\hline $\begin{array}{l}\text { Outcome } \\
\text { measure }\end{array}$ & $\begin{array}{l}\text { - Shoulder pain intensity (VAS, 0-10 NRS, validated shoulder-pain specific } \\
\text { questionnaire) } \\
\text { - Shoulder function (NRS, VAS, validated shoulder disability questionnaire). }\end{array}$ & $\begin{array}{l}\text { No baseline and follow-up data for either one of } \\
\text { these outcomes }\end{array}$ \\
\hline $\begin{array}{l}\text { Candidate } \\
\text { predictors }\end{array}$ & $\begin{array}{l}\text { At least one of the following potential predictors in addition to baseline levels } \\
\text { of the outcome measure (pain or disability): shoulder pain duration, sleep } \\
\text { disturbance due to shoulder pain, presence of weakness, cause of shoulder pain } \\
\text { (injury, or overuse due to work/hobbies), co-existing neck pain, psychosocial } \\
\text { complexity (fear-avoidance, catastrophizing, anxiety, depression, other), positive } \\
\text { expectations or preferences regarding treatment, presence of comorbidities. }\end{array}$ & \\
\hline $\begin{array}{l}\text { Length of } \\
\text { follow-up }\end{array}$ & Follow-up assessment at least 4 weeks after randomisation & $\begin{array}{l}\text { Trials including only very short-term follow-up (e.g. } \\
\text { after a single intervention session) }\end{array}$ \\
\hline Sample size & Minimum sample size at randomisation, 30 per intervention arm & \\
\hline
\end{tabular}


pain or diagnosed with subacromial conditions including rotator cuff tears, rotator cuff tendinopathy, subacromial impingement, or subacromial bursitis; (ii) frozen shoulder or adhesive capsulitis; (iii) glenohumeral osteoarthritis; and (iv) shoulder instability. The IPD meta-analysis will not include trials focusing on acute trauma (fractures, traumatic dislocations), inflammatory arthritis, shoulder pain resulting from cervical radiculopathy, or stroke-related shoulder pain.

\section{Interventions}

The IPD meta-analysis will focus on interventions that are commonly used in the primary care management of shoulder pain in the UK. Trials will be included that investigate the effectiveness of the following:

- Corticosteroid injection versus injection of an anaesthetic only or compared with control (advice and analgesics or no additional treatment)

- Physiotherapy-led exercise therapy (with/without manual therapy) compared with control (advice and analgesics or no additional treatment)

- Corticosteroid injection compared with physiotherapy-led exercise therapy (with/without manual therapy)

Primary care clinicians may also consider referral of patients with shoulder pain to secondary care services, offering more extensive treatments for shoulder pain. We will therefore also include trials investigating the effectiveness of the following:

- Interventions incorporating assessment and management of psychological risk factors, such as cognitive-behavioural approaches, or multimodal treatment programmes compared with exercise, injection, and/or control (advice and analgesics or no additional treatment)

- Surgical treatment compared with non-surgical treatment (exercise, injection) and/or control (advice and analgesics or sham surgery)

\section{Outcome measures}

We will include trials that have used (i) a measure of shoulder pain (e.g. visual analog scale (VAS), 0-10 NRS, validated shoulder-pain specific questionnaires) and/or (ii) a measure of shoulder pain related disability (NRS, VAS, validated shoulder disability questionnaires).

\section{Length of follow-up}

Trials will be included that have a follow-up assessment at least 4 weeks after randomisation. Outcomes will be analysed for up to 24 months after randomisation.

\section{Sample size}

In order to reduce the risk of small-study bias, we will only include trials with a sample size of at least 30 participants per treatment arm at the time of randomisation. This cut-point is arbitrary, but will ensure we obtain data for the largest trials. Furthermore, excluding the smallest trials will allow a more efficient approach to collecting data for this IPD meta-analysis, which is otherwise known to be very time-consuming [52]. Smallest trials are also more prone to baseline imbalance (by chance), and thus their exclusion will reduce the risk of identifying spurious treatment effects and treatmentpredictor interactions.

\section{Candidate predictors}

Developmental work has been conducted which has generated a shortlist of candidate predictors to be analysed in this IPD meta-analysis. A systematic review of randomised clinical trials identified 21 trials that investigated or suggested candidate predictors of the effects of treatment with corticosteroid injection, strengthening exercise and/or mobilisation, or advice and pain relief [53]. Only 7 trials included a moderation analysis or subgroup analysis, whereas 14 trials made untested suggestions of effect modification. The results of the systematic review informed a series of workshops with clinicians, who proposed profiles of patients most likely to respond to these treatments, and prioritised a list of 12 potential predictors. These 12 candidate predictors formed the basis of an international choice-based conjoint analysis survey [53], and most of these will be tested in the proposed IPD meta-analysis: baseline pain severity, baseline disability, sleep disturbance due to shoulder pain, presence of instability or weakness, cause of shoulder pain (injury or overuse due to work/hobbies), co-existing neck pain, psychosocial complexity (fear-avoidance, catastrophizing, anxiety, depression), positive expectations or preferences regarding treatment, and presence of comorbidities. Additionally, the author team suggested shoulder pain duration as an important candidate predictor of treatment effect.

\section{Patient and public involvement}

The perspectives of patients and clinicians involved in the management of shoulder pain be included in the design and reporting of the IPD meta-analysis, by involving an advisory group of people with experience of living with shoulder pain, and a clinical advisory group including physiotherapists, rheumatologists, GPs, and orthopaedic surgeons. Patient representatives will be invited from Keele University Research User Group. The advisory groups will be consulted regarding (i) candidate predictors and outcomes, (ii) interpretation and importance of findings to patients and clinicians, and (iii) how best 
to present the findings to the general public, patients, and clinicians.

\section{Searching and selection}

Potentially eligible trials were identified through existing relevant systematic reviews and an updated search of individual trials published after the search dates of these reviews (up until May 2018). Updated searches of electronic databases (restricted to randomised controlled trials) included MEDLINE, EMBASE, Cochrane Central Register of Controlled Trials (CENTRAL), PEDro, WHO ICTRP, and ClinicalTrials.gov. Table 2 presents the searches for MEDLINE (through Ovid) as an example; search terms were adapted for other databases. Citations will be imported into Covidence for screening and selection.

Two reviewers will screen titles and abstracts of trials identified from these searches against the eligibility criteria described above, excluding trials that clearly do not meet the eligibility criteria. Subsequently, full texts of trial reports will be retrieved and assessed for eligibility, again by two reviewers independently. Disagreements will be resolved through discussion or by third reviewer adjudication. Trial authors will be contacted if there are queries regarding eligibility.

\section{Extraction of aggregate data}

For each included trial, details on study design (randomisation and allocation procedure), study setting, sample size, baseline characteristics of the study sample (age, gender, diagnosis, duration and history of shoulder pain), details of interventions (type of treatment, duration, frequency, dose, co-interventions), comparator (advice, analgesics, other), candidate predictors, and outcome assessment (type of outcome measure, timing of followup, numbers lost to follow-up) will be extracted into tables. Two reviewers will independently extract outcome data on self-reported pain and disability at time points nearest to 6 weeks (short-term), 3-6 months (mediumterm), and $\geq 12$ months (long-term).

\section{Risk of bias assessment}

All trials identified from our searches will be assessed using the Cochrane Risk of Bias tool, regardless of whether they supply their IPD or not. Two researchers will independently grade risk of bias (unclear, high, or low risk of bias) based on sequence generation, allocation concealment, blinding of outcome assessor, incomplete outcome data, and selective outcome reporting. Although our aim is to obtain IPD from all studies, we recognise some teams may decline or no longer have access to data [54]. In this situation, we will also compare the risk of bias for those studies that do and do not provide their IPD. Studies providing their IPD will have a more thorough examination of their risk of bias, as aspects of design and conduct can be checked with the trial team, and the IPD allows an examination of some aspects to a greater detail (e.g. baseline balance and missing information) than possible with the study publication alone [55].

\section{Data transfer and quality assurance of individual participant data}

Principal investigators (PIs) or corresponding authors of identified trials will be contacted to inform them about the study and ask if they would be willing to share IPD. If there is no response, the institutes (e.g. head of department or research group) in which the trials have been performed will be contacted.

Those who express an interest in collaboration will be invited to read the protocol and requirements for sharing IPD will be discussed. The data custodian/representative of the institute who owns the data will then be invited to sign a data sharing agreement, which specifies the data requested, obligations, ownership of data, terms, authorship, and publications.

IPD requested from each trial will include the following:

- Baseline characteristics of participants (sociodemographic variables, characteristics of the shoulder condition, data regarding all candidate moderating variables)

- Randomisation code indicating random treatment allocation, any variables indicating non-adherence to protocol (protocol deviations), and duration and number of treatment sessions if these vary between individual participants

- Baseline and follow-up data regarding pain and disability outcomes at each time-point, where relevant including final scores for multi-item scales, and subscale scores for multidimensional scales (e.g. pain and function for SPADI). If available, EQ-5D will be requested as it contains items for pain and disability and may be used if trials have not included specific scales for pain or disability.

Trial authors will be asked to share relevant data regarding candidate predictors and outcomes regardless of whether such data has previously appeared in trial publications. The IPD received will not be used for any other research apart from that described in the data sharing agreement. All collaborators (one representative per trial) will be invited to be co-authors on manuscripts describing the principal analyses outlined in this protocol, subject to them meeting the International Committee of Medical Journal Editors criteria for authorship. 
Table 2 Searches to identify systematic reviews and potentially eligible trials (MEDLINE via Ovid)

\begin{tabular}{|c|c|}
\hline Systematic reviews & Randomised clinical trials \\
\hline 1. exp Shoulder Joint/ & 1. exp PAIN/ \\
\hline 2. shoulder.ti,ab. & 2. exp Shoulder Joint/ \\
\hline 3. "rotator cuff".ti,ab. & 3. 1 and 2 \\
\hline 4. glenohumeral.ti,ab. & 4. Shoulder Pain/ \\
\hline 5. subacromia\$.ti,ab. & 5. Shoulder Impingement Syndrome/ \\
\hline 6. or/1-6 & 6. exp Bursitis/ \\
\hline 7. Pain/ or pain.ti,ab. & 7. rotator cuff/ \\
\hline 8. 6 and 7 & $\begin{array}{l}\text { 8. ((shoulder or subacromial or glenohumeral or rotator cuff) } \\
\text { adj3 (instability or bursitis or frozen or impingement or tendinitis } \\
\text { or tendonitis or pain\$ or osteoarthr\$ or periarthriti\$)).ti,ab. }\end{array}$ \\
\hline 9. exp Shoulder Pain/ & 9. "adhesive capsulitis".ti,ab. \\
\hline 10. "adhesive capsulitis".ti,ab. & 10. or/3-9 \\
\hline 11. Shoulder Impingement Syndrome/ & 11. exp Adrenal Cortex Hormones/ \\
\hline 12. or/8-11 & 12. (steroid or corticosteroid).ti,ab. \\
\hline 13. Meta-Analysis as Topic/ & 13. 11 or 12 \\
\hline 14. meta analy\$.tw. & 14. inject\$.ti,ab. \\
\hline 15. metaanaly\$.tw. & 15.13 and 14 \\
\hline 16. Meta-Analysis/ & 16. exp Exercise/ or exp Exercise Therapy/ \\
\hline 17. (systematic adj (review\$1 or overview\$1)).tw. & 17. exercise\$.ti,ab. \\
\hline 18. exp Review Literature as Topic/ & 18. 16 or 17 \\
\hline 19. OR/13-18 & 19. exp Musculoskeletal Manipulations/ \\
\hline 20. cochrane.ab. & 20. ("manual therapy" or manipulation\$ or mobilisation\$).ti,ab. \\
\hline 21. embase.ab. & 21. (chiropract\$ or osteopath\$).ti,ab. \\
\hline 22. (psychlit or psyclit).ab. & 22. 19 or 20 or 21 \\
\hline 23. (psychinfo or psycinfo).ab. & 23. exp adaptation, psychological/ or exp behavior/ or exp psychology, social/ \\
\hline 24. (cinahl or cinhal).ab. & 24. (psychosocial or psycholog\$).ti,ab. \\
\hline 25. science citation index.ab. & 25. behavio\$.ti,ab. \\
\hline 26. bids.ab. & 26. 23 or 24 or 25 \\
\hline 27. cancerlit.ab. & 27. (surgery or surgical or operat\$).ti,ab. \\
\hline 28. "web of science".ab. & 28. exp orthopedic procedures/ or orthopedics/ \\
\hline 29. or/20-28 & 29. 27 or 28 \\
\hline 30. reference list\$.ab. & 30. (physio\$ or physical therap\$).ti,ab. \\
\hline 31. bibliograph\$.ab. & 31. exp Physical Therapy Modalities/ \\
\hline 32. hand-search\$.ab. & 32. 30 or 31 \\
\hline 33. relevant journals.ab. & 33. 15 or 18 or 22 or 26 or 29 or 32 \\
\hline 34. manual search\$.ab. & 34. 10 and 33 \\
\hline 35. or $30-34$ & 35. random\$.ti,ab. \\
\hline 36. selection criteria.ab. & 36. factorial\$.ti,ab. \\
\hline 37. data extraction.ab. & 37. crossover\$.ti,ab. \\
\hline 38. 36 or 37 & 38. cross over\$.ti,ab. \\
\hline 39. Review/ & 39. placebo\$.ti,ab. \\
\hline 40. 66 and 67 & 40. (doub\$ adj blind\$).ti,ab. \\
\hline 41. Comment/ & 41. (sing\$ adj blind\$).ti,ab. \\
\hline 42. Letter/ & 42. assign\$.ti,ab. \\
\hline 43. Editorial/ & 43. allocat\$.ti,ab. \\
\hline
\end{tabular}


Table 2 Searches to identify systematic reviews and potentially eligible trials (MEDLINE via Ovid) (Continued)

\begin{tabular}{ll}
\hline Systematic reviews & Randomised clinical trials \\
\hline 44. animal/ & 44. volunteer\$.ti,ab. \\
45. human/ & 45. double-blind procedure/ \\
46. 44 not (44 and 45$)$ & 46. crossover-procedure/ \\
47. 41 or 42 or 43 or 46 & 47. randomized controlled trial/ \\
48. 19 or 29 or 35 or 40 & 48. single-blind procedure/ \\
49. 48 not 47 & 49. or/35-48 \\
50.12 and 49 & 50.34 and 49 \\
\hline
\end{tabular}

Collaborators will be invited to send their anonymised dataset as an encrypted file to the host institution, where this will be stored on a secure server. Prior to data transfer, there will be a process to verify that the person receiving the data is the accurate and intended recipient for the data. Any additional processes or requirements for data transfer stipulated by the collaborating institutions will be adhered to. Datasets will be accepted in any form, provided that all data are anonymised and variables and categories are adequately labelled in English. However, ideally, the format will be a two-dimensional spreadsheet with one participant per row and variables listed in columns and different time points on separate spreadsheets.

Datasets will be examined for missing and unusual values, with any issues resolved through communication with the original authors. We will attempt to reproduce the results included in each initial trial publication, including baseline characteristics and self-reported pain and disability at a time point nearest to 6 weeks, 3-6 months, and 12 months. Discrepancies or missing information will be discussed and clarified with original trial authors, where possible requesting analysis scripts. Following satisfactory data checking, each dataset will be converted to a common format and variables will be renamed in a consistent manner. The original scales of outcomes and covariate measurements that are reported will be utilised, where possible. To ensure compatibility across studies, when required, attempts will be made to convert variables to the same scale for all studies, by seeking to standardise the scale for outcomes and covariate measurements, for example, converting continuous outcomes to a standardised (e.g. $\mathrm{N}(0,1))$ scale, by subtracting individual outcome values by the mean in the same study and then dividing by the standard deviation of values across individuals in the same study. Once each study data is cleaned and standardised, individual trial datasets will then be combined to form a new master dataset with a variable added to indicate the original trial.

\section{Approaches to dealing with missing participant-level data in trials}

Participant-level missing data on candidate predictors and individual outcomes will be described. Under a 'missing-at-random' assumption, and where repeated outcome measures are available over time, individuals with partially missing outcome data (e.g. at some timepoints) will be included in analyses (without imputation) using a longitudinal data (multi-level) modelling framework. Otherwise, they will be excluded, as this does not bias results for trials assuming the outcomes are missing at random [56]. If there is a considerable amount of missing baseline data for candidate predictors or covariates of interest (e.g. age, gender), this will be handled using multiple imputation, for those variables where a 'missing-at-random' assumption is deemed appropriate. Subsequently, for each meta-analysis, Rubin's rule will be used to combine meta-analysis results across the imputed datasets [57].

\section{Analysis}

\section{General approach to the IPD meta-analysis}

The IPD meta-analysis will be conducted in two steps: (1) estimating overall treatment effects on shoulder pain and disability outcomes for each of the treatment comparisons listed in objectives 1 and, and (2) estimating treatment-predictor interactions within each of the comparisons and separately for each of the candidate predictors (objective 3). We expect few trials will include data on all candidate predictors, and therefore, it is not our intention to develop a full prediction model that would consider all candidate predictors in a single model. Each of the candidate predictors will be separately tested for effect modification, using all trial datasets that have provided sufficient data on the treatment comparison, candidate predictor, and outcome measure.

For both steps, the IPD meta-analysis can be conducted using either a one-stage or two-stage approach [58]. Both approaches will be used in this IPD metaanalysis, with the two-stage approach being conducted as a sensitivity analysis. A two-stage approach is often preferred, as this approach automatically ensures that clustering of participants within trials is accounted for and aggregation bias can be avoided, which is more likely in a one-stage approach $[59,60]$. This approach also allows the use of the Hartung-Knapp correction to 
inflate confidence intervals, accounting for the uncertainty of the estimated between-study variance [61]. However, the one-stage approach is likely to be more practical for modelling of non-linear associations of candidate predictors with outcome. Therefore, the analysis will be conducted using the one-stage approach (hierarchical model which will include both study-level and patient-level covariates in the same model), if possible using the Kenward-Roger method to inflate confidence intervals to account more fully for the uncertainty of variance estimates. Clustering will be accounted for by using a separate intercept per study and with separate residual variance and adjustment terms per study. Random treatment effects and treatment-predictor interaction terms will be included to allow for potential heterogeneity. Candidate predictors will be centred by their mean to avoid ecological bias [62]. A two-stage analysis will be performed as a sensitivity analysis to check whether conclusions are consistent.

\section{Outcome measures}

All analyses will be conducted for (i) severity of shoulder pain, measured using either a $0-10$ numerical rating scale, 0-100 VAS, or pain scale (e.g. SPADI pain subscale), and (ii) level of shoulder function, measured using a validated shoulder disability questionnaire, with trials contributing to the analysis if they have provided sufficient data on any one of these measures.

\section{IPD meta-analysis models}

All analyses will be carried out using Stata 15 [63] or SAS 9.3 [64]. All analyses will be performed based on an intention-to-treat principle. All models will allow for random effects and will be estimated using restricted maximum likelihood (REML).

For both outcomes (shoulder pain and disability scores), the IPD meta-analysis will utilise a linear regression framework, where the final pain/disability score will be regressed against the baseline score, the treatment effect, the candidate predictor, and the interaction term. If studies use different pain or disability scales, these will be converted to a common scale if possible or otherwise estimates will be expressed as standardised mean difference. If standardised scales are required, the standardised final score will be regressed and the standardised baseline score adjusted for. For trials that contained multiple centres or a cluster-design, the hierarchical model will handle the clustering appropriately. Continuous candidate predictors will be entered as a linear term primarily, but sensitivity analysis will examine if nonlinear trends are more plausible, using restricted cubic splines [65].

Adjustment for age and gender, as well as baseline pain/disability score, will be made in all analyses [66]. If there are repeated follow-up scores, the model will be adapted to include a repeated measures model where all follow-up times are jointly analysed, and the correlation among repeated measures from the same participants accounted for [67]. This approach can naturally handle missing follow-up scores for some patients, under a missing at random assumption. Treatment-predictor interactions may vary between time points, which will be an important criterion when interpreting statistically significant interaction terms $(p<0.10)$. Consistent evidence across multiple time points will add credence to it being a genuine (causal) predictor of treatment response [68]. Any model convergence problems will be reported clearly.

\section{Data presentation}

All summary and study-specific results will be presented in tables and via forest plots, with heterogeneity disseminated by $I$-squared, estimates of between-study variance and approximate $95 \%$ prediction intervals [69]. The entire process with be reported according to the PRISMAIPD guidelines [70]. Overall treatment effects and treatment effects for each relevant subgroup (where significant treatment-predictor interactions will be identified) will be produced.

\section{Small-study effects}

Small-study effects refer to systematic differences in the effects from bigger and smaller studies and may occur due to many reasons, such as publication bias, heterogeneity, and availability bias (i.e. non-participation of trials toward the IPD meta-analysis). As mentioned, we aim to reduce the risk of small study bias by only including trials with a sample size of at least 30 participants per arm at the time of randomisation. However, in metaanalyses of 10 trials or more, small-study effects (which may highlight the potential for publication bias and availability bias) will be examined using contourenhanced funnel plots and tests for asymmetry such as Egger's test and Peter's test [71]. In the presence of fewer than 10 trials, there is low power to detect small-study effects [72].

\section{Sensitivity analyses}

Sensitivity analyses will be carried out to assess the influence of important methodological factors and assess the robustness of the results of the main analysis. Sensitivity analyses will be based on (i) exclusion of trials studies at high risk of bias, (ii) use of restricted cubic splines to model potential non-linear associations for candidate predictors measured on a continuous scale, and (iii) the use of a two-stage IPD meta-analysis framework. For the latter, in the first stage, each trial providing IPD will be analysed separately to provide interaction estimates 
between each candidate predictor and treatment effect. Then, in the second stage, the interaction estimates will be synthesised using a random-effects meta-analysis, to produce a summary interaction estimate for each candidate predictor. REML will be used to estimate the random-effects meta-analysis models, with 95\% confidence intervals for summary effects derived using the Hartung-Knapp approach to account for uncertainty in the estimated variance terms [73, 74]. Overall treatment effects and treatment effects for each subgroup will be produced (where significant treatment-predictor interactions are identified). For non-linear interactions, the second stage will use a multivariate meta-analysis model [75].

\section{Acknowledgements}

Not applicable

\section{Authors' contributions}

DvdW, DBu, $\mathrm{MH}$, and RR designed the study with significant contributions from GJvdH, JW, CH, DBe, KB, LC, CL, and DR. OB, CM, CL, GWJ, GM, LC, and DvdW contributed to the literature searches, study identification, and initial risk of bias assessment. DvdW, DBu, MH, and RR prepared the manuscript, which was critically reviewed and approved by all authors. RR is the guarantor of the IPD meta-analysis.

\section{Funding}

This paper presents independent research funded by the National Institute for Health Research (NIHR) under its Programme Grants for Applied Research Programme (Reference Number RP-PG-0615-20002), with co-funding from Arthritis Research UK. DBu was funded by an NIHR School for Primary Care Research Post-Doctoral Fellowship, and developmental work by CM was funded by a doctoral fellowship of the NIHR School for Primary Care Research. The funders will play no role in the analysis or interpretation of this meta-analysis. The views expressed are those of the author(s) and not necessarily those of the NHS, the $\mathrm{NIHR}$, or the Department of Health and Social Care.

\section{Availability of data and materials}

Not applicable

\section{Ethics approval and consent to participate}

No new data will be collected and only anonymised data will be used, and therefore, this study is exempted from research ethical or governance approval $[76,77]$. Dissemination will include open access publication of findings in high-impact academic journals and presentation at (inter) national academic and professional conferences. Additionally, a dedicated website, our NHS partnerships and links to professional bodies, guideline development organisations (e.g. NICE), the Academic Health Sciences Networks (AHSN), and campaigning and education networks of patient organisations will be used to disseminate the research findings. A patient advisory group will be involved in the interpretation and dissemination of findings and assist the team to develop plain English summaries for news websites, newspapers, and other relevant media outlets.

\section{Consent for publication}

Not applicable

\section{Competing interests}

GW-J sits on the NICE Guideline Review Panel "Workplace health: Long-term sickness absence and capability for work". CL receives payment for courses on the assessment and treatment of rotator cuff related shoulder pain.

\section{Author details}

${ }^{1}$ Institute for Primary Care and Health Sciences, Arthritis Research UK Primary Care Centre, Centre for Prognosis Research, Keele University, Keele, UK. ${ }^{2}$ School of Health Sciences, University of Liverpool, Liverpool, UK. ${ }^{3}$ Department of Social Dentistry, Academic Center for Dentistry Amsterdam, University of Amsterdam and Vrije Universiteit Amsterdam, Amsterdam, The
Netherlands. ${ }^{4}$ Department of General Practice and Elderly Care Medicine, University Medical Centre Groningen, Groningen, The Netherlands. ${ }^{5}$ San Antonio Military Medical Center, Fort Sam Houston, San Antonio, TX, USA. ${ }^{6}$ Centre for Health, Exercise and Sports Medicine, Department of Physiotherapy, University of Melbourne, Melbourne, Australia. ${ }^{7}$ Haywood Academic Rheumatology Centre, Staffordshire and Stoke-on-Trent Partnership Trust, Stoke-on-Trent, UK. ${ }^{8}$ Nuffield Department of Primary Care and Health Science, University of Oxford, Oxford, UK. ${ }^{9}$ Nuffield Department of Orthopaedics, Rheumatology and Musculoskeletal Sciences (NDORMS), University of Oxford, Oxford, UK.

Received: 16 January 2019 Accepted: 16 May 2019

Published online: 08 August 2019

\section{References}

1. GBD 2016 Disease and Injury Incidence and Prevalence Collaborators. Global, regional, and national incidence, prevalence, and years lived with disability for 328 diseases and injuries for 195 countries, 1990-2016: a systematic analysis for the Global Burden of Disease Study 2016. Lancet. 2017;390(1):1211-59.

2. Luime JJ, Koes BW, Hendriksen IJ, Burdorf A, Verhagen AP, Miedema HS, Verhaar JA. Prevalence and incidence of shoulder pain in the general population; a systematic review. Scand J Rheumatol. 2004;33(2):73-81.

3. Greving K, Dorrestijn O, Winters JC, Groenhof F, van der Meer K, Stevens M, Diercks RL. Incidence, prevalence, and consultation rates of shoulder complaints in general practice. Scand J Rheumatol. 2012;41(2):150-5.

4. Jordan KP, Kadam UT, Hayward R, Porcheret M, Young C, Croft P. Annual consultation prevalence of regional musculoskeletal problems in primary care: an observational study. BMC Musculoskelet Disord. 2010;11:144.

5. Watson J, Helliwell P, Morton V, Adebajo A, Dickson J, Russell I, Torgerson D. Shoulder acute pain in primary healthcare: is retraining effective for GP principals? SAPPHIRE--a randomized controlled trial. Rheumatology (Oxford). 2008;47(12):1795-802.

6. Virta $L$, Joranger $\mathrm{P}$, Brox Jl, Eriksson R. Costs of shoulder pain and resource use in primary health care: a cost-of-illness study in Sweden. BMC Musculoskelet Disord. 2012;13:17.

7. Oh LS, Wolf BR, Hall MP, Levy BA, Marx RG. Indications for rotator cuff repair: a systematic review. Clin Orthop Relat Res. 2007;455:52-63.

8. Littlewood C, Ashton J, Chance-Larsen K, May S, Sturrock B. Exercise for rotator cuff tendinopathy: a systematic review. Physiotherapy. 2012;98(2): 101-9.

9. Dong W, Goost H, Lin XB, Burger C, Paul C, Wang ZL, Zhang TY, Jiang ZC, Welle K, Kabir K. Treatments for shoulder impingement syndrome: a PRISMA systematic review and network meta-analysis. Medicine (Baltimore). 2015; 94(10):e510.

10. Zheng X, Li K, Wei Y, Tie H, Yi X, Huang W. Nonsteroidal anti-inflammatory drugs versus corticosteroid for treatment of shoulder pain: a systematic review and meta-analysis. Arch Phys Med Rehabil. 2014;95:1824-31.

11. Song A, Higgins LD, Newman J, Jain NB. Glenohumeral corticosteroid injections in adhesive capsulitis: a systematic search and review. PM R. 2014; 6(12):1143-56.

12. Page MJ, Green S, Kramer S, Johnston RV, McBain B, Chau M, Buchbinder R. Manual therapy and exercise for adhesive capsulitis (frozen shoulder). Cochrane Database Syst Rev. 2014;8:CD011275.

13. Page MJ, Green S, McBain B, Surace SJ, Deitch J, Lyttle N, Mrocki MA, Buchbinder R. Manual therapy and exercise for rotator cuff disease. Cochrane Database Syst Rev. Issue 6. Art. No.: CD012224. https://doi.org/10. 1002/14651858.CD012224.

14. Abdulla SY, Southerst D, Côté P, Shearer HM, Sutton D, Randhawa K, Varatharajan S, Wong JJ, Yu H, Marchand AA, Chrobak K, Woitzik E, Shergill Y, Ferguson B, Stupar M, Nordin M, Jacobs C, Mior S, Carroll LJ, van der Velde G, Taylor-Vaisey A. Is exercise effective for the management of subacromial impingement syndrome and other soft tissue injuries of the shoulder? A systematic review by the Ontario Protocol for Traffic Injury Management (OPTIMa) Collaboration. Man Ther. 2015;20(5):646-56.

15. Ellegaard K, Christensen R, Rosager S, Bartholdy C, Torp-Pedersen S, Bandholm T, Danneskiold-Samsøe B, Bliddal H, Henriksen M. Exercise therapy after ultrasound-guided corticosteroid injections in patients with subacromial pain syndrome: a randomized controlled trial. Arthritis Res Ther. 2016;18(1):129. 
16. Mintken PE, McDevitt AW, Cleland JA, Boyles RE, Beardslee AR, Burns SA, Haberl MD, Hinrichs LA, Michener LA. Cervicothoracic manual therapy plus exercise therapy versus exercise therapy alone in the management of individuals with shoulder pain: a multicenter randomized controlled trial. J Orthop Sports Phys Ther. 2016;46(8):617-28.

17. Rhon DI, Boyles RB, Cleland JA. One-year outcome of subacromial corticosteroid injection compared with manual physical therapy for the management of the unilateral shoulder impingement syndrome: a pragmatic randomized trial. Ann Intern Med. 2014;161(3):161-9.

18. Holt TA, Mant D, Carr A, Gwilym S, Beard D, Toms C, Yu LM, Rees J. Corticosteroid injection for shoulder pain: single-blind randomized pilot trial in primary care. Trials. 2013;14:425.

19. Judge A, Murphy RJ, Maxwell R, Arden NK, Carr AJ. Temporal trends and geographical variation in the use of subacromial decompression and rotator cuff repair of the shoulder in England. Bone Joint J. 2014;96(1):70-4.

20. Holmgren T, Bjornsson Hallgren H, Oberg B, Adolfsson L, Johansson K. Effect of specific exercise strategy on need for surgery in patients with subacromial impingement syndrome: randomised controlled study. BMJ. 2012;344:e787.

21. Ketola S, Lehtinen J, Arnala I, Nissinen $M$, Westenius $H$, Sintonen $H$, Aronen P, Konttinen YT, Malmivaara A, Rousi T. Does arthroscopic acromioplasty provide any additional value in the treatment of shoulder impingement syndrome? A two-year randomised controlled trial. J Bone Joint Surg. 2009; 91:1326-34

22. Carr AJ, Cooper CD, Campbell MK, Rees JL, Moser J, Beard DJ, Fitzpatrick R, Gray A, Dawson J, Murphy J, Bruhn H, Cooper D, Ramsay CR. Clinical effectiveness and cost-effectiveness of open and arthroscopic rotator cuff repair [the UK Rotator Cuff Surgery (UKUFF) randomised trial]. Health Technol Assess. 2015;19(80):1-218.

23. Beard DJ, Rees JL, Cook JA, Rombach I, Cooper C, Merritt N, Shirkey BA, Donovan JL, Gwilym S, Savulescu J, Moser J, Gray A, Jepson M, Tracey I, Judge A, Wartolowska K, Carr AJ. CSAW Study Group. Arthroscopic subacromial decompression for subacromial shoulder pain (CSAW): a multicentre, pragmatic, parallel group, placebo-controlled, three-group, randomised surgical trial. Lancet. 2018;391(10118):329-38. https://doi.org/10. 1016/S0140-6736(17)32457-1

24. Schrøder CP, Skare $\varnothing$, Reikerås $O$, Mowinckel $P$, Brox Jl. Sham surgery versus labral repair or biceps tenodesis for type II SLAP lesions of the shoulder: a three-armed randomised clinical trial. Br J Sports Med. 2017;51(24):1759-66.

25. Paavola M, Malmivaara A, Taimela S, Kanto K, Inkinen J, Kalske J, Sinisaari I, Savolainen V, Ranstam J, TLN J. Finnish Subacromial Impingement Arthroscopy Controlled Trial (FIMPACT) Investigators. Subacromial decompression versus diagnostic arthroscopy for shoulder impingement: randomised, placebo surgery controlled clinical trial. BMJ. 2018;362:k2860.

26. Hanchard LM, Handoll HH, Takwoingi Y. Physical tests for shoulder impingements and local lesions of bursa, tendon or labrum that may accompany impingement. Cochrane Database Syst Rev. 2013;4:CD007427.

27. Alqunaee M, Galvin R, Fahey T. Diagnostic accuracy of clinical tests for subacromial impingement syndrome: a systematic review and metaanalysis. Arch Phys Med Rehabil. 2012;93(2):229-36.

28. Hegedus EJ, Goode AP, Cook CE, Michener L, Myer CA, Myer DM, Wright AA. Which physical examination tests provide clinicians with the most value when examining the shoulder? Update of a systematic review with metaanalysis of individual tests. Br J Sports Med. 2012;46(14):964-78.

29. Miller-Spoto M, Gombatto SP. Diagnostic labels assigned to patients with orthopedic conditions and the influence of the label on selection of interventions: a qualitative study of orthopaedic clinical specialists. Phys Ther. 2014;94(6):776-91.

30. Ottenheijm RP, Hesselmans NJ, Kemper A, Moser A, de Bie RA, Dinant GJ, Cals J. GPs' perspectives on the diagnostic work-up in patients with shoulder pain: a qualitative study. Eval Clin Pract. 2014;20(3):239-45.

31. Buchbinder R, Staples MP, Shanahan EM, Roos JF. General practitioner management of shoulder pain in comparison with rheumatologist expectation of care and best evidence: an Australian national survey. PLoS One. 2013 Apr 16;8(4):e61243.

32. Artus M, van der Windt DA, Afolabi EK, Buchbinder R, Chesterton LS, Hall A, Roddy E, Foster NE. Management of shoulder pain by UK general practitioners (GPs): a national survey. BMJ Open. 2017;7(6):e015711.

33. Laslett M, Steele M, Hing W, McNair P, Cadogan A. Shoulder pain patients in primary care - part 1: clinical outcomes over 12 months following standardized diagnostic workup, corticosteroid injections, and communitybased care. J Rehabil Med. 2014;46(9):898-907.
34. Van der Windt DAWM, Koes BW, Boeke AJP, Devillé WLJM, De Jong BA, Bouter LM. Shoulder disorders in general practice: prognostic indicators of outcome. Br J Gen Pract. 1996;46:519-23.

35. Kuijpers T, Van der Windt DAWM, Boeke AJP, Twisk JWR, Vergouwe $Y$, Bouter LM, Van der Heijden GJMG. Clinical prediction rules for the prognosis of shoulder pain in general practice. Pain. 2006;120:276-85.

36. Bot SDM, Van der Waal JM, Terwee CB, Van der Windt DAWM, Scholten RJPM, Bouter LM, Dekker J. Predictors of outcome in neck and shoulder symptoms: a cohort study in general practice. Spine. 2005;30:459-70.

37. Masters S, O'Doherty L, Mitchell GK, Yelland M. Acute shoulder pain in primary care - an observational study. Aust Fam Physician. 2007;36(6):473-6.

38. Keijsers E, Feleus A, Miedema HS, Koes BW, Bierma-Zeinstra SM. Psychosocial factors predicted nonrecovery in both specific and nonspecific diagnoses at arm, neck, and shoulder. J Clin Epidemiol. 2010;63(12):1370-9.

39. Laslett M, Steele M, Hing W, McNair P, Cadogan A. Shoulder pain in primary care - part 2: predictors of clinical outcome to 12 months. J Rehabil Med. 2015;47:66-71.

40. Engebretsen $\mathrm{K}$, Grotle M, Bautz-Holter E, Ekeberg OM, Brox Jl. Predictors of shoulder pain and disability index (SPADI) and work status after 1 year in patients with subacromial shoulder pain. BMC Musculoskelet Disord. 2010;11:218.

41. Roh YH, Lee BK, Noh JH, Oh JH, Gong HS, Baek GH. Effect of depressive symptoms on perceived disability in patients with chronic shoulder pain. Arch Orthop Trauma Surg. 2012;132(9):1251-7.

42. Chester R, Jerosch-Herold C, Lewis J, Shepstone L. Psychological factors are associated with the outcome of physiotherapy for people with shoulder pain: a multicentre longitudinal cohort study. Br J Sports Med. 2018;52(4): 269-75. https://doi.org/10.1136/bjsports-2016-096084

43. Rasmussen-Bar Rasmussen-Barr E, Grooten WJ, Hallqvist J, Holm LW, Skillgate E. Are job strain and sleep disturbances prognostic factors for neck/shoulder/arm pain? A cohort study of a general population of working age in Sweden. BMJ Open. 2014;4(7):e005103.

44. Kromer TO, Sieben JM, de Bie RA, Bastiaenen $\mathrm{CH}$. Influence of fearavoidance beliefs on disability in patients with subacromial shoulder pain in primary care: a secondary analysis. Phys Ther. 2014;94(12):1775-84.

45. Sindhu BS, Lehman LA, Tarima S, Bishop MD, Hart DL, Klein MR, Shivakoti M, Wang YC. Influence of fear-avoidance beliefs on functional status outcomes for people with musculoskeletal conditions of the shoulder. Phys Ther. 2012; 92(8):992-1005.

46. George SZ, Parr JJ, Wallace MR, Wu SS, Borsa PA, Dai Y, Fillingim RB. 2014 inflammatory genes and psychological factors predict induced shoulder pain phenotype. Med Sci Sports Exerc. 2014;46(10):1871-81.

47. Van der Windt DAWM, Kuijpers T, Jellema P, van der Heijden GJ, Bouter LM. Do psychological factors predict outcome in both low back pain and shoulder pain? Ann Rheum Dis. 2007;66:313-9.

48. Reilingh M, Kuijpers T, Tanja A, Van der Windt DA. Course and prognosis of shoulder symptoms in general practice. Rheumatology. 2008;47(5):724-30.

49. Jones S, Hanchard N, Hamilton S, Rangan A. A qualitative study of patients' perceptions and priorities when living with primary frozen shoulder. BMJ Open. 2013;3(9):e003452.

50. James Lind Alliance Priority Setting Partnerships. Surgery for common shoulder problems. http://www.jla.nihr.ac.uk/priority-setting-partnerships/ surgery-for-common-shoulder-problems (last accessed: 22nd January 2018).

51. Chester R, Shepstone L, Daniell H, Sweeting D, Lewis J, Jerosch-Herold C. Predicting response to physiotherapy treatment for musculoskeletal shoulder pain: a systematic review. BMC Musculoskelet Disord. 2013;14:203.

52. Altman DG, Trivella M, Pezzella F, Harris AL, Pastorino U. Systematic review of multiple studies of prognosis: the feasibility of obtaining individual patient data. In: Auget J-L, Balakrishnan N, Mesbah M, Molenberghs G, editors. Advances in statistical methods for the health sciences. Boston: Birkhäuser; 2006. p. 3-18.

53. McRobert C. Primary care decision making for shoulder pain: identifying treatment effect moderators using clinical expertise (PhD thesis). Keele University; 2018.

54. Ahmed I, Sutton AJ, Riley RD. Assessment of publication bias, selection bias and unavailable data in meta-analyses using individual participant data: a database survey. BMJ. 2012;344:d7762.

55. Tierney JF, Vale C, Riley R, et al. Individual participant data (IPD) metaanalyses of randomised controlled trials: guidance on their use. PLoS Med. 2015;12:e1001855.

56. Sterne JA, White IR, Carlin JB, Spratt M, Royston P, Kenward MG, Wood AM, Carpenter JR. Multiple imputation for missing data in epidemiological and clinical research: potential and pitfalls. BMJ. 2009;338:b2393. 
57. Carpenter JR, Kenward M. Multiple imputation and its application. Chichester: Wiley; 2013.

58. Burke DL, Ensor J, Riley RD. Meta-analysis using individual participant data: one-stage and two-stage approaches, and why they may differ. Stat Med. 2017;36(5):855-75.

59. Riley RD, Lambert PC, Abo-Zaid G. Meta-analysis of individual participant data: rationale, conduct, and reporting. BMJ. 2010;340:c221.

60. Fisher DJ, Copas AJ, Tierney JF, Parmar MK. A critical review of methods for the assessment of patient-level interactions in individual participant data meta-analysis of randomized trials, and guidance for practitioners. J Clin Epidemiol. 2011;64(9):949-67.

61. Hartung J, Knapp G. A refined method for the meta-analysis of controlled clinical trials with binary outcome. Stat Med. 2001;20:3875-89.

62. Hua H, Burke DL, Crowther MJ, Ensor J, Tudur Smith C, Riley RD. One-stage individual participant data meta-analysis models: estimation of treatmentcovariate interactions must avoid ecological bias by separating out withintrial and across-trial information. Stat Med. 2017:36:772-89.

63. StataCorp. Stata Statistical Software: Release 15. College Station: StataCorp LP; 2018.

64. SAS Institute Inc. SAS/STAT_ 9.3 user's guide. Cary, NC: SAS Institute Inc; 2011.

65. Gasparrini A, Armstrong B, Kenward MG. Multivariate meta-analysis for nonlinear and other multi-parameter associations. Stat Med. 2012;31:3821-39.

66. Riley RD, Kauser I, Bland M, Thijs L, Staessen JA, Wang J, Gueyffier F, Deeks JJ. 2013 meta-analysis of randomised trials with a continuous outcome according to baseline imbalance and availability of individual participant data. Stat Med. 2013;32(16):2747-66.

67. Jones AP, Riley RD, Williamson PR, Whitehead A. Meta-analysis of individual patient data versus aggregate data from longitudinal clinical trials. Clin Trials. 2009;6(1):16-27.

68. Sun X, Briel M, Walter SD, Guyatt GH. Is a subgroup effect believable? Updating criteria to evaluate the credibility of subgroup analyses. BMJ. 2010; 340:c117. https://doi.org/10.1136/bmj.c117.

69. Riley RD, Higgins JP, Deeks JJ. Interpretation of random effects metaanalyses. BMJ. 2011;342:d549.

70. Stewart LA, Clarke M, Rovers M, Riley RD, Simmonds M, Stewart G, Tierney JF. PRISMA-IPD Development Group. Preferred Reporting Items for Systematic Review and Meta-Analyses of individual participant data: the PRISMA-IPD statement. JAMA. 2015;313(16):1657-65.

71. The Cochrane Collaboration. In: JPT H, Green S, editors. Cochrane handbook for systematic reviews of interventions version 5.1.0; 2011. www.handbook. cochrane.org (accessed 5 May 2017).

72. Sterne JA, Sutton AJ, loannidis JP, et al. Recommendations for examining and interpreting funnel plot asymmetry in meta-analyses of randomised controlled trials. BMJ. 2011;343:d4002.103.

73. Cornell JE, Mulrow CD, Localio R, Stack CB, Meibohm AR, Guallar E, Goodman SN. Random-effects meta-analysis of inconsistent effects: a time for change. Ann Intern Med. 2014;160(4):267-70.

74. IntHout J, loannidis JP, Borm GF. The Hartung-Knapp-Sidik-Jonkman method for random effects meta-analysis is straightforward and considerably outperforms the standard DerSimonian-Laird method. BMC Med Res Methodol. 2014;14:25.

75. Riley RD, Price MJ, Jackson D, et al. Multivariate meta-analysis using individual participant data. Res Synth Method. 2015;6:157-74.

76. Taichman DB, Backus J, Baethge C, et al. Sharing clinical trial data: a proposal from the International Committee of Medical Journal Editors. JAMA. 2016;315:467-8.

77. Menikoff J. Letter from Jerry Menikoff, MD, JD. Director, Office for Human Research Protections, to ICMJE Secretariat. 2017. http://icmje.org/news-andeditorials/menikoff_icmje_questions_20170307.pdf. Accessed 22 Jan 2018.

\section{Publisher's Note}

Springer Nature remains neutral with regard to jurisdictional claims in published maps and institutional affiliations.

\section{Ready to submit your research? Choose BMC and benefit from}

- fast, convenient online submission

- thorough peer review by experienced researchers in your field

- rapid publication on acceptance

- support for research data, including large and complex data types

- gold Open Access which fosters wider collaboration and increased citations

- maximum visibility for your research: over $100 \mathrm{M}$ website views per year

At BMC, research is always in progress.

Learn more biomedcentral.com/submissions 